\title{
NOTES ON NEW WORLD SAPROPHYTES II
}

\author{
Compiled by P. J. M. MAAS
}

Instituut voor Systematische Plantkunde, Postbus 80.102, 3508 TC Utrecht

As a result of a revision of the neotropical saprophytes (Triuridaceae, Burmanniaceae, and Gentianaceae) several new species have to be described and some new combinations have to be made.

1. Sciaphila polygyna Maas sp. nov.

Herba saprophytica, gracilis. Caulis simplex, $16-17 \mathrm{~cm}$ altus, $0,1-0,5 \mathrm{~mm}$ diametro. Rhizoma ignota. Folia squamiformia, $1-5$, ad 1,5 mm longa. Inflorescentia racemosa, vaga, 3-13 cm longa, 1-1,3 cm lata, (20-) 30-65-flora. Bracteae anguste triangulares, 1-2 mm longae, fusco-striatae. Pedicelli rubri, gracillimi, 5-8 mm longi, juventute ascendentes, mox horizontaliter patentes, apice in fructo recurvati. Flores unisexuales, monoecii, 50-55 inferiores feminei, 10-15 superiores masculi. Flores masculi ca $2 \mathrm{~mm}$ diametro; tepala 6 , anguste triangularia, mox reflexa, $0,6-0,7 \mathrm{~mm}$ longa, apice juventute dense barbata. Stamina 3, filamentis ca. $0.2 \mathrm{~mm}$ longis, antheris $0,2 \times 0,4 \mathrm{~mm}$. Flores feminei ca 2 $\mathrm{mm}$ diametro; tepala similia floribus masculis Carpella ca 30 , late obovidea, $0,1-0,2 \mathrm{~mm}$ longa. Stylus $0,2-0,3 \mathrm{~min}$ longus, ovario multo superans, apice dense penicillato-papillosus. Capsula lata obovoidea ad subglobosa, 0,7-0,9 $\times 0,5-0,6$ $\mathrm{mm}$, glabra, seminibus ellipsoideis ad obovoideis, $0,5-0,7 \mathrm{~mm}$ longis.

TYPE. Haught 1725 (holotype, US), Camp Carare V, vicinity of Puerto Berrio, between Ríos Carare and Magdalena, 100-700 m, Santander, Colombia.

Distribution. Colombia and French Guiana; forest.

French Guiana. Without locality, Perrottet sn, anno 1821 (P).

This species is very well distinguished from any other neotropical species of Sciaphila by its extremely high number of female flowers (50-55).

2. Sciaphila oligantha Maas sp. nov. Fig. 1 .

Herba saprophytica. Caulis albus, simplex, $15-25 \mathrm{~cm}$ altus, $0,5-1 \mathrm{~mm}$ diametro. Rhizoma longe repens, ad $1 \mathrm{~mm}$ diametro, radicibus filiformibus, ad $15 \mathrm{~cm}$ longis et $1 \mathrm{~mm}$ crassis, pilis albis, erectis, dense obtectis. Folia squamiformia, $3-5$, anguste triangularia, $3-4 \mathrm{~mm}$ longa. Inflorescentia racemosa, vaga, $5-10 \mathrm{~cm}$ longa, 2-3,5 cm lata, 10-15-flora. Bracteae anguste triangulares, $2,5-5 \mathrm{~mm}$ longae. Pedicelli 7-18 mm longi, horizontaliter patentes, apice leviter recurvati. Flores unisexuales, monoecii, albi, 2-6 inferiores feminei, 5-9 suberioribus masculi. Flores masculi 3-4 mm diametro; tepala 4-6, triangulari-ovata, mox reflexa, 1,5-2 mm longa, intus praesertim versus apicem dense papillosa. Stamina 
3, filamentis $0,5-0,7 \mathrm{~mm}$ longis, antheris $0,4 \times 0,7 \mathrm{~mm}$. Flores feminei ca $6 \mathrm{~mm}$ diametro; tepala 5-6, anguste triangularia, mox reflexa, 2,5-2,7 mm longa, intus praesertim versus apicem dense papillosa. Carpella ca 50, late obovoidea, $0,4-0,6 \mathrm{~mm}$ longa, praesertim versus apicem dense papillosa. Stylus ca $0,5 \mathrm{~mm}$ longus, ovario paulo superans, parte stigmatico deltoideo, dense penicillatopapillosa, seminibus ellipsodeis ad anguste ellipsoideis, 1,8-2 $\mathrm{mm}$ longis.

TYPE. Prance et al. 9025 (holotype, INPA), Reserva Florestal Ducke, $27 \mathrm{~km}$ of Manaus-Itacoatiara Road, Amazonas, Brazil.

Distribution. Restricted to the neighbourhood of Manaus, Amazonas, Brazil; forest on terra firme.

Brazil. Amazonas: Reserva Florestal Ducke, Picada P.E.P. 41, Rodrigues 585 (paratype, INPA).

This species is closely related to $S$. albescens, from which it differs by its longer pedicels, its lower number of flowers, and its much shorter style. Just as the recently described S. rubra (MAAS 1979) it is endemic to the Ducke Forest Reserve and Egler Forest Reserve (where I observed it personally in September 1977) near Manaus.

MaAS, P. J. M. 1979. Notes on New World Saprophytes I. Triuridaceae. Acta Bot. Neerl. 28 : 89.

II. BURMANNIACEAE (P. J. M. MAAS, J. VAN BENTHEM, and H. C. SNELDERS)

\section{Burmannia jonkeri Van Benthem \& Maas sp. nov. Fig. 2.}

Herba non-saprophytica, simplex vel interdum ramosa, $6-20 \mathrm{~cm}$ alta. Folia saepe purpurea, anguste triangulari-ovata ad anguste ovata, 1,3-3,9 $(-5,0) \mathrm{mm}$ longa. Flores 1-5, terminales, 2-5 mm inter se remoti. Bracteae saepe purpureae, anguste ellipticae ad anguste ovatae, $(1,6-) 2-3,5(-4,5) \mathrm{mm}$ longae. Flores purpurei ad caerulei, sessiles vel breviter pedicellati, $12-18 \mathrm{~mm}$ longi. Tepala exterioria radiata, anguste ovata ad ovata, 4,0-6,4(-7,3) $\mathrm{mm}$ longa, intus saepe papillosa. Tepala interioria anguste triangulari-ovata ad anguste ellitipca, $0,8-1,5 \mathrm{~mm}$ longa, intus saepe papillosa. Tubus perianthii trigonus, trialatus, $4,0-5,5(-7,1) \mathrm{mm}$ longus, alis semi-obovatis ad semi-ellipticis, $4,2-9,6 \mathrm{~mm}$ longis et $(0,4-) 0,9-2,1 \mathrm{~mm}$ latis. Stamina sessilia, connectivo trapeziformi, $0,2-0,4 \mathrm{~mm}$ longo, apice appendicibus duobus triangularibus, $0,25-0,50 \mathrm{~mm}$ longis et basi appendice obtriangulari, $0,25-0,50 \mathrm{~mm}$ longo munito, thecis $0,3-0,7 \mathrm{~mm}$ longis. Stylus 4,7-5,5 mm longus. Ovarium anguste obovoideum ad anguste obconicum, 3,5-7,1 mm longum. Capsula 5,0-6,2 mm longa, seminibus conicis ad ellipsoideis, $0,17-0,25 \mathrm{~mm}$ longis.

TYPE. Irwin et al. 12827 (holotype, UB; isotypes, K, NY, U) c. $15 \mathrm{~km} \mathrm{~W}$ of Veadeiros, Chapada dos Veadeiros, Goiás, Brazil.

Distribution. Central Brazil, the states of Goiás and Mato Grosso.

Brazil. Mato Grosso: Serra Ricardo Franco, alt. 800 m, Windisch 1597, 1685,1946 (U, paratypes). 
This species is closely related to $B$. alba and $B$. grandiflora.

It is a great pleasure to dedicate it to Prof. F. P. Jonker who was the first to monograph Burmanniaceae, and who stimulated us to start this additional study of the family.

\section{Burmannia vaupesana Van Benthem \& Maas sp. nov. Fig. 3.}

Herba non-saprophytica, simplex vel rare ramosa, 8-30 cm alta. Folia basalia anguste triangularia, folia caulina anguste triangulari-ovata ad ovata, $1,7-4,7(-6,7) \mathrm{mm}$ longa. Inflorescentia cincinnata, bifida, cincinnibus $2-9$ floris, 3-17 mm longis, floribus 0,7-2,5 mm inter se remotis. Bracteae ellipticae ad anguste ovatae, 1,4-3,5 mm longae. Flores purpurei ad albo-virides, 4,5-9 mm longi, sessiles. Tepala exterioria late ad transverse ovata, $(0,9-) 1,1-1,5 \mathrm{~mm}$ longa, marginibus involutis. Tepala interioria elliptica ad spathulata, $(0,4-), 6-0,9 \mathrm{~mm}$ longa, apice leviter incurvato. Tubus perianthii trigonus, $1,5-2,2 \mathrm{~mm}$ longus, late trialatus, alis late semiturbinatis ad late semi-ellipticis, 1-4 mm longis, 0,7-2,3 mm latis. Stamina sessilia, connectivo trapeziformi, $0,15-0,30 \mathrm{~mm}$ longo, apice appendicibus duobus, ellipticis ad spathulatis, $0,2-0,4 \mathrm{~mm}$ longis et basi appendice late obtriangulari, $0,1-0,2 \mathrm{~mm}$ longo munito thecis $0,3-0,5 \mathrm{~mm}$ longis. Stylus $2-2,7 \mathrm{~mm}$ longus. Ovarium anguste ellipsoideum ad anguste obconicum, 2-6 mm longum. Capsula 2,2-6 mm longa, seminibus ellipsoideis ad ovoideis, $0,15-0,25 \mathrm{~mm}$ longis.

TYPE. Zarucchi 2226 (holotype, U; isotypes, A, COL, F, GH, INPA, K, MO, NY, P, RB, S, UC, US, VEN, Z), savanna at major rapids, Río Parana-Pichuna, Mitú and vicinity, Vaupés, Colombia.

Distribution. Colombia, the regions of Vaupés and Amazonas; in savannas.

Colombia. Amazonas: Río Caquetá, Araracuara, Maguire et al. 14119 (U). Vaupés: Río Kuduyari, Cerro de Yapoboda, Garay 102 (COL), Schultes \& Cabrera 14227 (COL, U, US); Río Cubiyu, Humbert \& Schultes 23711 (P), Schultes \& Cabrera 18350 (U, UC); Río Parana-Pichuna, Schultes \& Cabrera 19961 (U, US); Mitú, lower Río Kubuyu, Zarucchi 2185, 2186 (A, U); Mitú, Rio Parana-Pichuna, Zarucchi 1949 (A, U).

The relationship of this species is rather obscure, although it superficially looks very much like the saprophytic $B$. tenella. I am greatly indebted to Jim Zarucchi for providing a fantastic type collection.

3. Gymnosiphon recurvatus Snelders \& Maas sp. nov. Fig. 4.

Herba saprophytica, 4-25 cm alta. Caulis albus (ad purpureus) simplex vel ramosus. Folia squamiformia, ovata ad late ovata, 1,2-2,3 mm longa. Inflorescentia bifida, 3-18-flora, cincinnibus 0,6-4 cm longis. Bracteae ovatae ad late ovatae, 1.6-2 mm longae. Pedicelli mox recurvati, 0,8-2,5 mm longi. Flores albi, 7,5-8,4 mm longi. Tepala exterioria trilobata, $2,1-3,4 \mathrm{~mm}$ longa. Tepala interioria ovata, 0,4-0,6 mm longa. Tubus perianthii, 4,4-5,4 mm longus, leviter costatus. Stamina $0,4-0,6 \mathrm{~mm}$ sub insertione tepalorum interiorum inserta, 
connectivo late obovoideo, 0,3-0,4 mm longo. Stylus (stigmate incluso) $3,5-3,7$ $\mathrm{mm}$ longus, stigmatibus exappendiculatis. Ovarium transverse ellipsoideum, 1-1,2 mm longum, glandulis globosis, $0,4-0,5 \mathrm{~mm}$ diametro. Capsula globosa ad transverse ellipsoidea, ad $2,7 \mathrm{~mm}$ longa, leviter costata, seminibus late ellipsoideis, $0,5-0,9 \mathrm{~mm}$ longis.

TYPE. P. J. M. \& H. Maas \& R. Boyan 2530 (holotype, U + alc; isotypes, BBS, COL, F, K, MO, NY, P, UG, US, VEN), Mazaruni River, 1-6 km N of Kamarang, alt. $450-550 \mathrm{~m}$, rain forest on lateritic gravel, Guyana.

DistRIBUTION. Restricted to forests around Kamarang, Guyana. Locally very common; between 450 and $800 \mathrm{~m}$.

GuYaNA. Mt. Latipú, $15 \mathrm{~km} \mathrm{~N}$ of Kamarang, Maas et al. 2634 (paratypes, U, UG); Kamarang, Maas et al. 2685 (paratype, U, alc.), 4153 (paratypes, CAY, K, NY, S, U, UG, Z).

This species is closely related to another guianan species, $G$. guianensis, but it is readily distinguished by its recurved pedicels, and in the absence of wings on the floral tube.

4. Gymnosiphon minutus Snelders \& Maas sp. nov.

Herba saprophytica, minuta, $3-7,5 \mathrm{~cm}$ alta. Caulis albus ad purpureus, simplex vel ramosus. Folia squamiformia, ovata ad late ovata, $(0,7-) 1,1-2,0 \mathrm{~mm}$ longa. Inflorescentia bifida, 1-4-flora, cincinnibus 0,2-2,5 cm longis. Bractea anguste ovatae ad ovatae, (1,6-)2,3-4 mm longae, marginibus involutis. Pedicelli saepe absentes vel ad $1 \mathrm{~mm}$ longi. Flores albi, erecti, 5,2-8 $\mathrm{mm}$ longi. Tepala exterioria trilobata, alba (in alabastro lutea), $1,6-2,2 \mathrm{~mm}$ longa. Tepala interioria ovata, ca. $0,17 \mathrm{~mm}$ longa. Tubus perianthii $4,3-6 \mathrm{~mm}$ longus. Stamina $0,5 \mathrm{~mm}$ sub insertione tepalorum interiorum inserta, connectivo obovoideo, $0,2-0,3 \mathrm{~mm}$ longo. Stylus (stigmate incluso) 2,1-2,6 mm longus, stigmatibus exappendiculatis. Ovarium obconicum ad late obovoideum, 1-2 mm longum, glandulis globosis, 0,25-0,30 mm diametro. Capsula late obovoidea ad obovoidea, ad 3,2 $\mathrm{mm}$ longa, seminibus late ellipsoideis, $0,18-0,25 \mathrm{~mm}$ longis.

TYPE. Maas \& Mc. Alpin 1469 (holotype, U, alc.), Esquinas Forest, prov. Puntarenas, Costa Rica.

Distribution. Costa Rica, Colombia, Venezuela, Brazil, and Guyana; in forests from $15-250 \mathrm{~m}$.

Colombia. Amazonas-Vaupés: Mouth of Río Pacoa, Schultes \& Cabrera 13050 (US). Sur de Santander: Barranca Bermeja, Haught 1434 (US).

Venezuela. Amazonas: Cerro Yapacana, Steyermark \& Bunting 103016 (VEN).

GuYana. NW-District, Kwabanna, Maas et al. 2413, 2493 (U, UG); Mazaruni Station, Tutin 79 (BM).

BrazIL. Amazonas: Serra Curicuriari, Prance, Maas et al. 16162 (NY).

5. Gymnosiphon brachycephalus Snelders \& Maas sp. nov. Fig. 5.

Herba saprophytica, minuta, 5-8 cm alta. Caulus albus, simplex vel ramosus. 
Folia squamiformia, ovata ad late ovata, $1,2-2,1 \mathrm{~mm}$ longa. Inflorescentia capitata, bifida, $0,2-0,5 \mathrm{~cm}$ longa, 2-6-flora. Bracteae ovatae ad late ovatae, $1,8-3,5 \mathrm{~mm}$ longae. Pedicelli absentes vel ad $0,2(-0,6) \mathrm{mm}$ longi. Flores albi, 6,2-6,6 mm longi. Tepala exterioria trilobata, 1,1-1,6 mm longa. Tepala interioria ovata, ca. $0,15 \mathrm{~mm}$ longa. Tubus perianthii $4,6-5 \mathrm{~mm}$ longus. Stamina ca. $0,6 \mathrm{~mm}$ sub insertione tepalorum interiorum inserta, connectivo late ovoideo, ca. 0,2 mm longo. Stylus (stigmate incluso) $2,4-3 \mathrm{~mm}$ longus, stigmatibus exappendiculatis. Ovarium late ellipsoideum, 1,7-2,2 mm longum, glandulis globosis, ca. $0,2 \mathrm{~mm}$ diametro. Capsula late ovoidea ad globosa, usque ad 2,4 mm longa, seminibus late ellipsoideis ad globosis, $0,25-0,30 \mathrm{~mm}$ longis.

TYPE. P. J. M. \& H. Maas \& R. Boyan 2686 (holotype, U), Mora-forest near Kamarang, Mazaruni R., alt. 450-550 m, Guyana.

DisTRIBUTION. Guyana and Suriname; rain forest.

GUYANA. Bartica-Potaro Rd., greenheart-wallaba forest near Barbara Creek, Sandwith 1135 (paratype, K).

Suriname. $6.5 \mathrm{~km}$ NNW. of Tafelberg-airstrip, Hoogmoed \& Polder S-6 (paratype, $\mathrm{U}$, alc.).

This species is related to G. minutus.

6a. Dictyostega orobanchioides (Hooker) Miers ssp. parviflora (Bentham) Snelders \& Maas comb. nov.

Basionym: Dictyostega schomburgkii Miers var. parviflora Bentham in HooKER, J. Bot. 7: 13.1855.

TYPE. Spruce 2623 (holotype, K; isotypes, BM, BR, P), Rio Vaupés, Amazonas, Brazil.

6b. Dictyostega orobanchioides (Hooker) Miers ssp. purdieana (Bentham) Snelders \& Maas comb. nov.

Basionym: Dictyostega purdieana Bentham in HooKer, J. Bot. 7: 14, 1855. TYPE. Purdie s. n. (holotype, K), Maracay, dep. Magdalena, Colombia.

III. GENTIANACEAE (P. J. M. MAAS and P. RUYTERS)

1. Voyria kupperi (Suessenguth) Ruyters \& Maas comb. nov.

Basionym: Leiphaimos kupperi Suessenguth in FEDDE, Repert. 42: 46. 1937. TYPE. Kupper 417 (holotype, M), Siquirres, prov. Limón, alt. 300 m, Costa Rica.

2. Voyria parasitica (Schldl. \& Chamisso) Ruyters \& Maas comb. nov.

Basionym: Leiphaimos parasitica Schldl. \& Chamisso. Linnaea 6: 387, 1831. TYPE. Schiede s.n. (holotype, HALLE), Papantla, Mexico. 
3. Voyria corymbosa Splitgerber var. alba (Standley) Ruyters \& Maas comb. nov. Basionym: Leiphaimos albus Standley, Contr. U. S. Nat. Herb. 20: 198. 1919. TyPE. Pittier 4295 (holotype. US), Puerto Obaldia, San Blas Coast, alt. 0-50 m, Panama. 

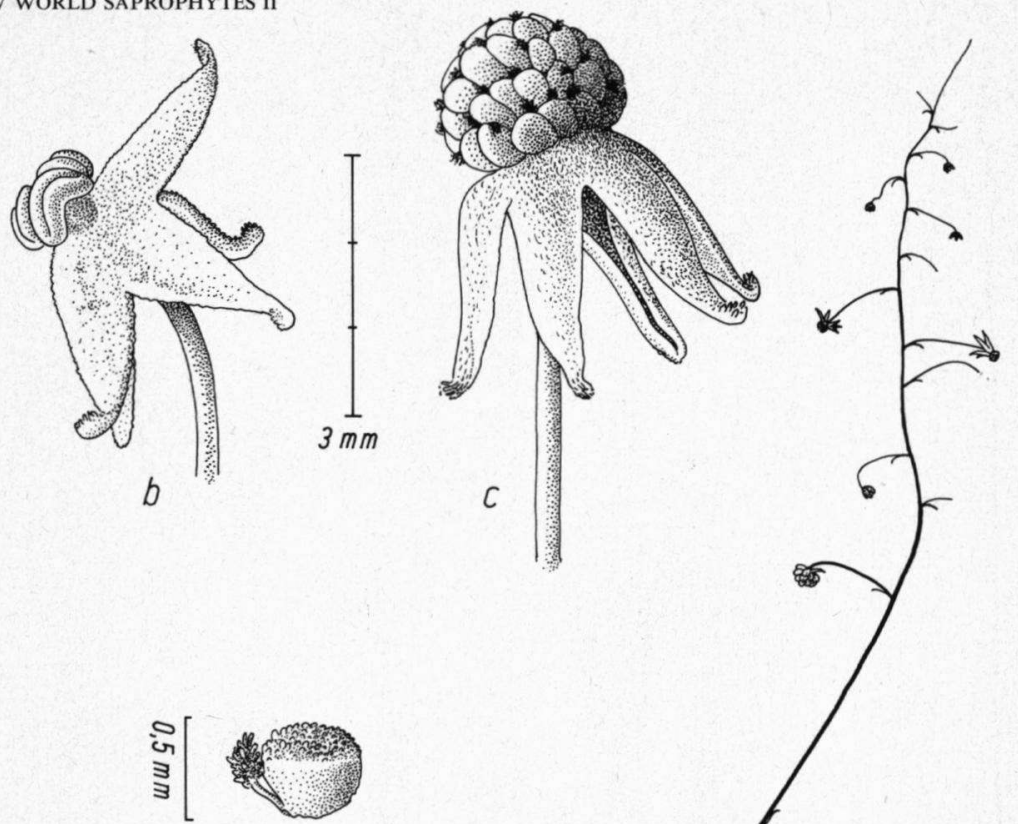

d
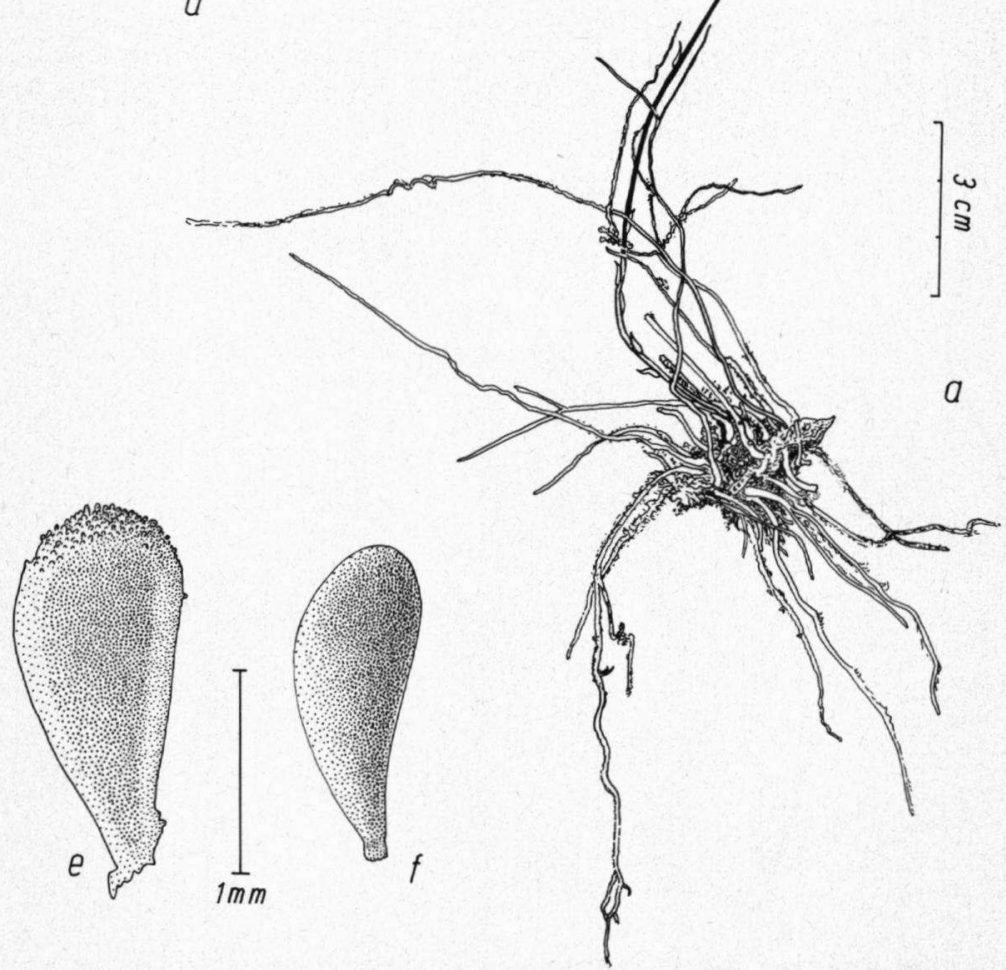

$a$

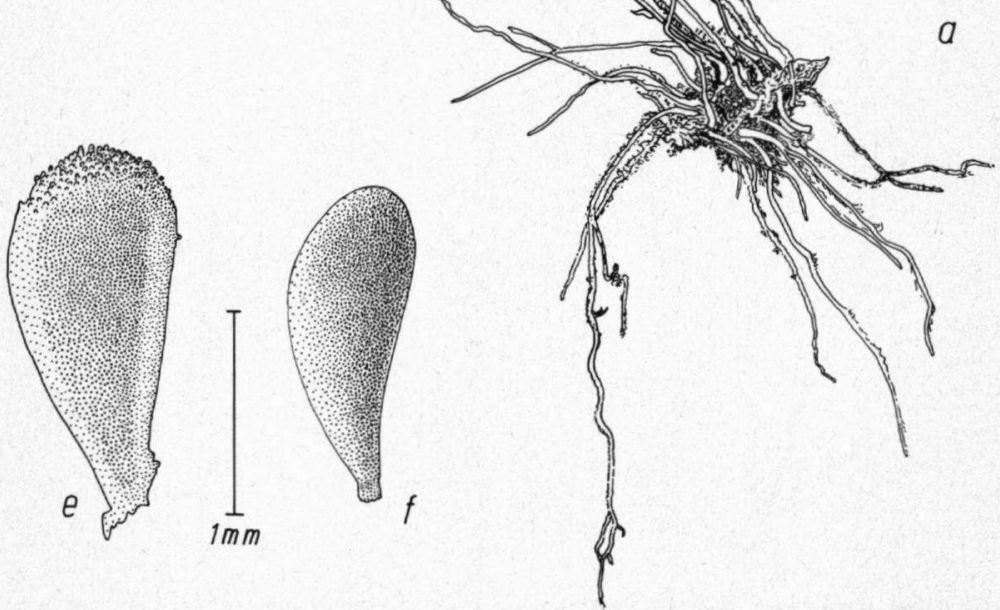

$\omega$$$
x^{2}
$$

Fig. 1. Sciaphila oligantha (a, c-f, Prance et al. 9025 ; b, Rodrigues 585). a. habit, b. male flower, c. female flower, d. ovary with style and stigma, e. fruit, f. seed. 

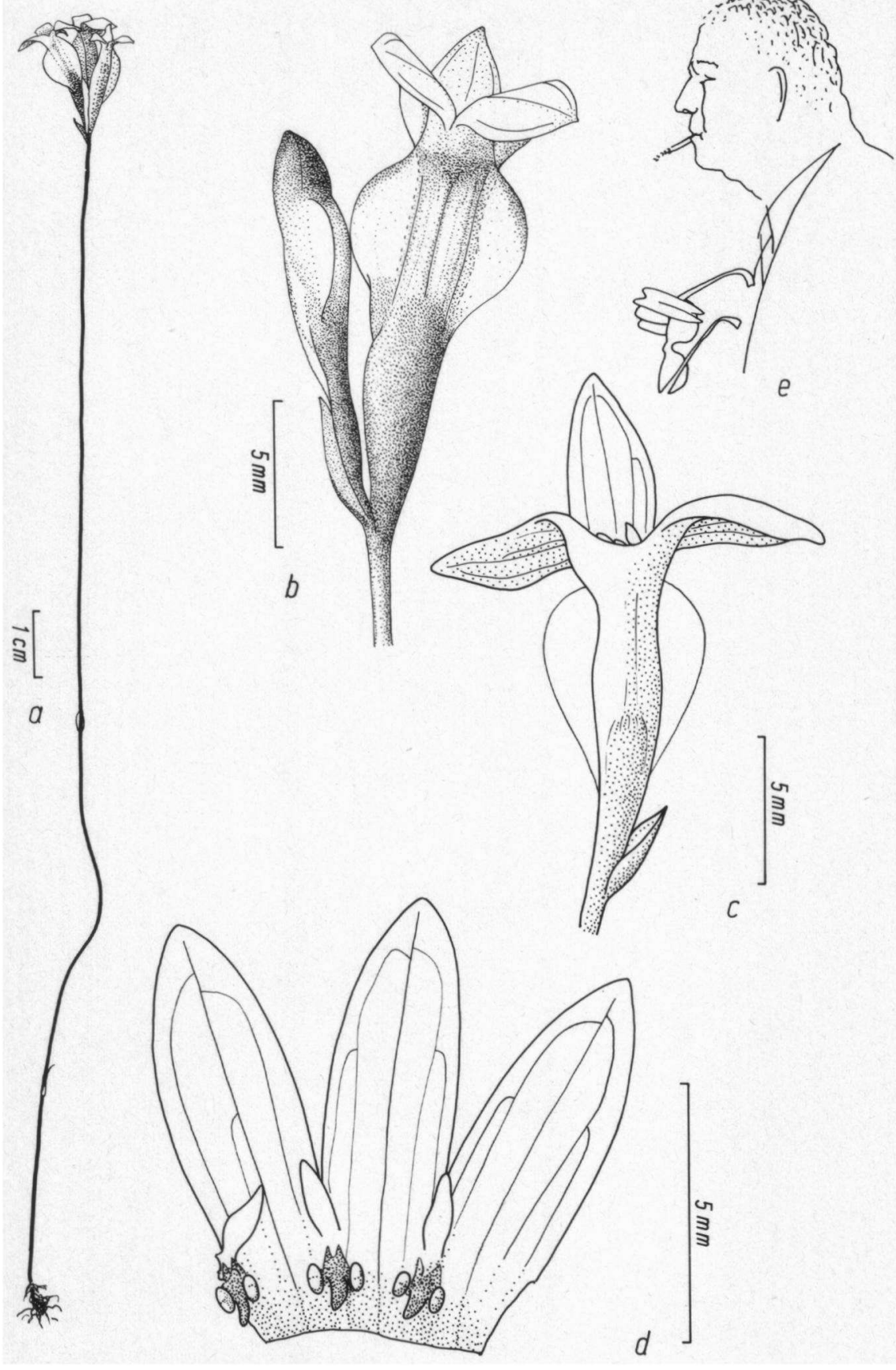

Fig. 2. Burmannia jonkeri (a-d, Irwin et al. 12827). a. habit, b. part of inflorescence, c. flower, d. dissected flower, showing inner and outer tepals, and stamens, e. F. P. Jonker. 


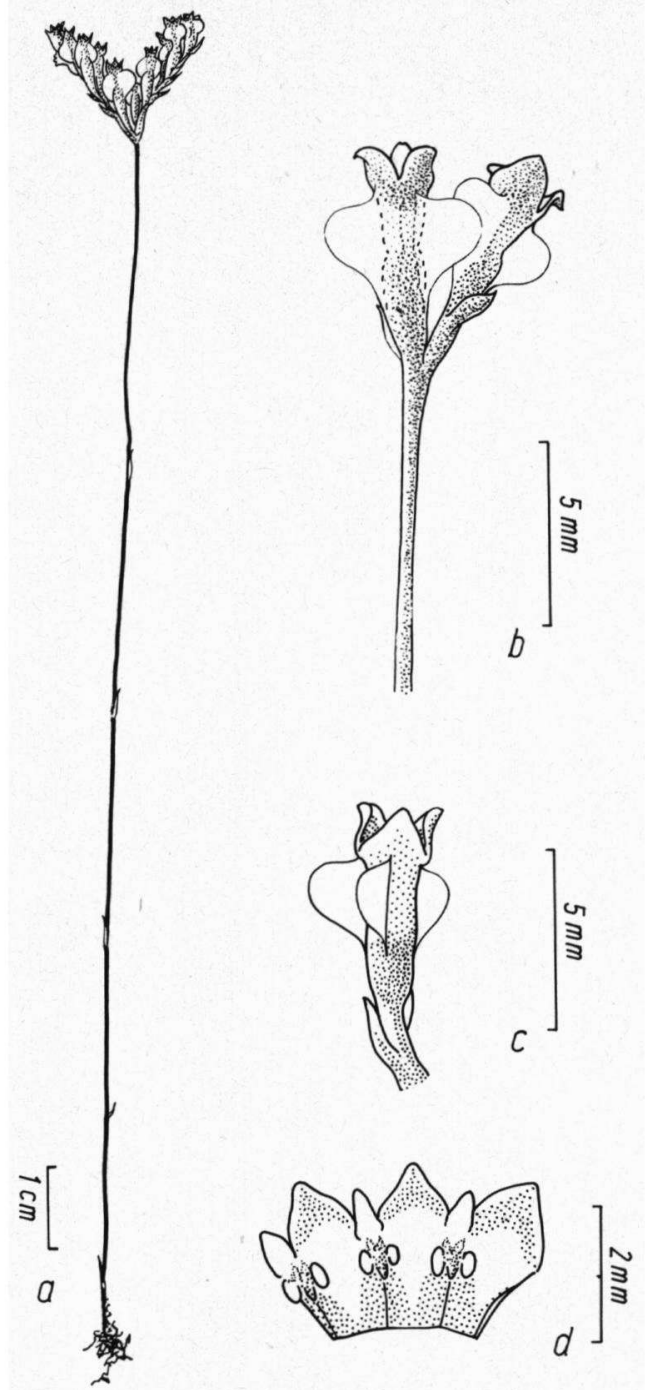

Fig. 3. Burmannia vaupesana (a, Zarucchi 1949; b-d, Zarucchi 2226). a.habit, b. upper part of stem, c. flower, d. dissected flower, showing inner and outer tepals, and stamens. 


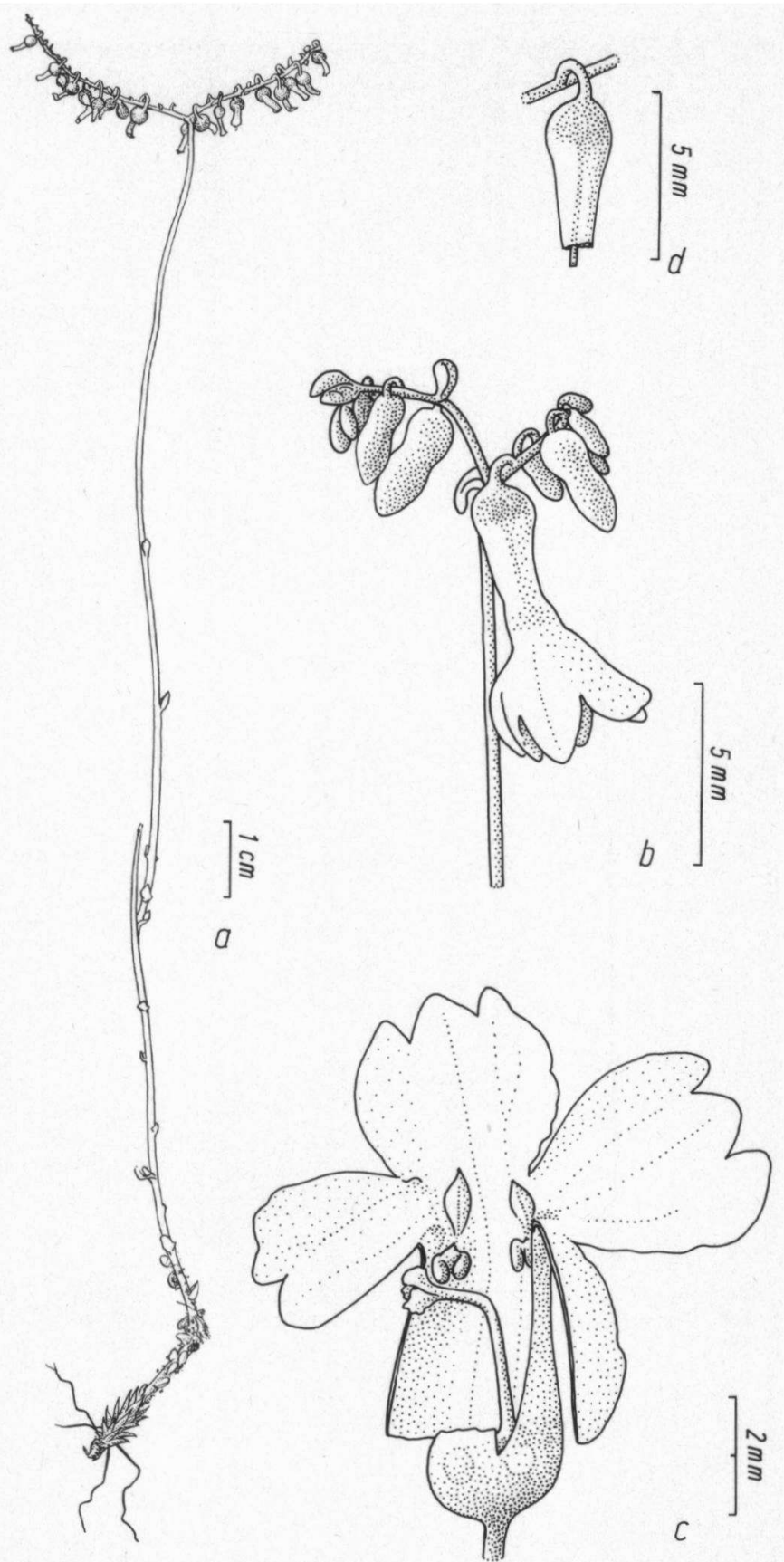

Fig. 4. Gymnosiphon recurvatus (a-d, Maas et al. 2530). a. habit, b. young inflorescence, showing recurved floral buds, c. dissected flower, showing inner and outer tepals, stamens, style, and stigma. 


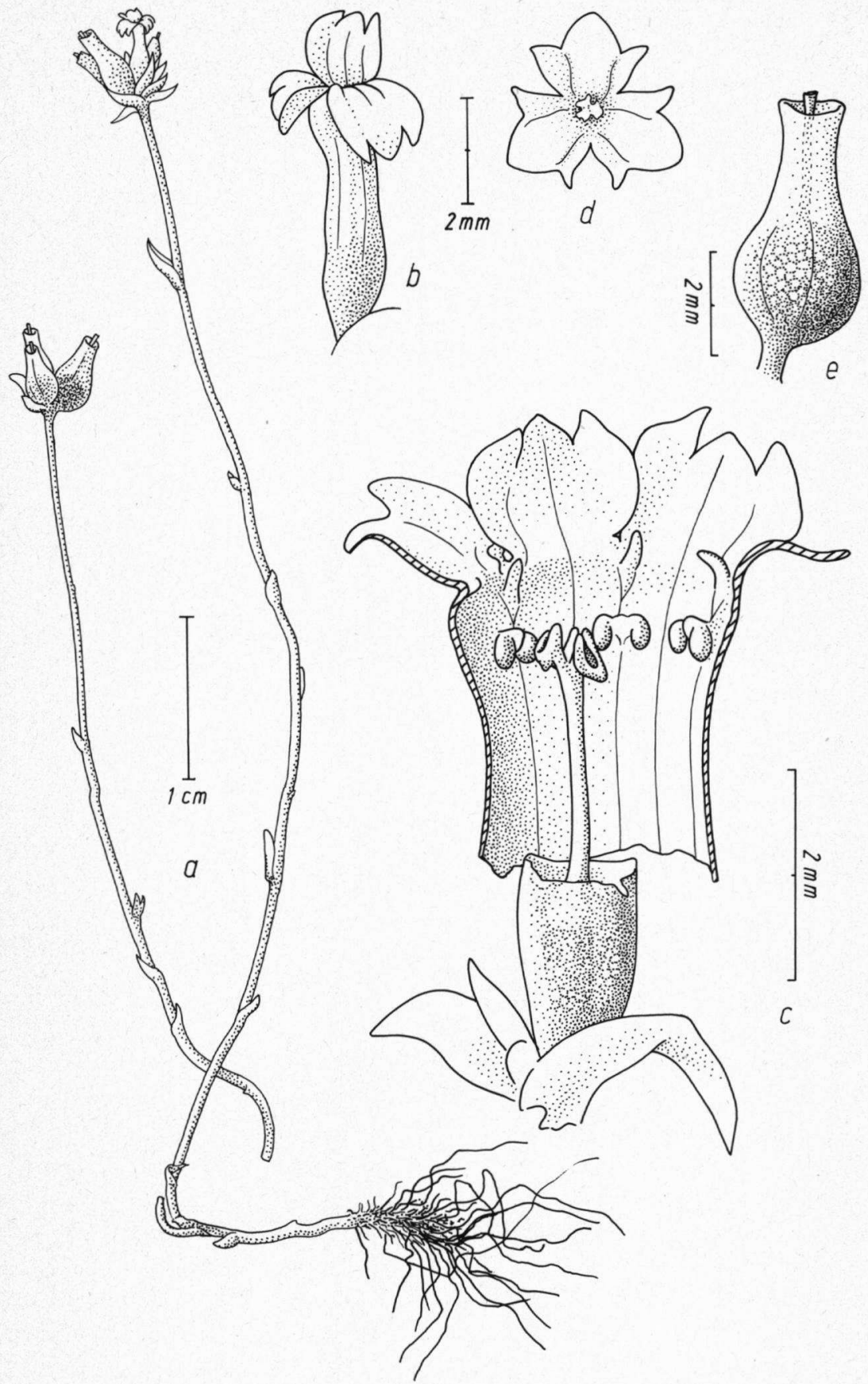

Fig. 5. Gymnosiphon brachycephalus (a-e, Maas et al. 2686). a. habit (two plants), b. flower, c. dissected flower, showing inner and outer tepals, stamens, style, and stigma, d. flower seen from above, e. young fruit. 